\title{
COMPAGNIE IBERICHE A MILANO
} NEL SECONDO QUATTROCENTO

\author{
Patrizia MaInoni \\ Universitd degli Studi \\ Milano (Italia)
}

\section{L'analisi dell'attività di mercanti forestieri nelle diverse aree dell'} Europa medievale.e un tema di consolidata tradizione, che vanta una bibliografia imponente. Meno puntuale è invece stata la considerazione prestata, oltre che alle motivazioni propriamente economiche, alle interconnessioni fra potere politico e imprese mercantili, specie nei suoi legami con la finanza pubblica, che sono state studiate soprattutto in riferimento alle vicende di singole aziende o personalità ${ }^{2}$. In questo contesto anche presenze numericamente scarse possono risultare significative, perché indice di un mutamento di rapporti non solamente commerciali, anche se le "sperimentazioni" in campo mercantile costituiscono un aspetto caratteristico delle iniziative economiche fra medioevo e rinascimento.

Sino al secondo Quattrocento non si ha notizia di interessi diretti dei mercanti catalani in Lombardia, mentre invece e bene conosciuto l' interscambio condotto soprattutto per il tramite di Genova e, a partire dalla fine del Trecento, attraverso la presenza degli operatori provenienti dallo

'Un modello del genero a ancora Richard DE ROOVER, The rise and decline of the Medici Bank, New York, 1966; au figure di singoli operatori, a mero titolo di esempio, ai possono citare Melisea Meriem BUUARD, Filippo Strozzi and the Medici. Fovor \& Finance in Sixteenth Censury Florence \& Rome, Cambridge, 1980, e Michel MOUAT, Jacques Cocur ou l'esprit d'entreprise aus XVe sizcle, Paris, 1988. Rocentemente il tema delle dlites mercantili in Europa a stato oggetto di convegni i cui risultati sono poi confluiti in volumi: v. Sistemi di rapporti ed elises economiche (secoli XII-XVII) a cura di Mario DEI TREPPO, Napoli, 1994 (Quademi di Europe mediterranee, 8) o Economia e politica melle cirrd europee dei secoli XIIXVI, a cura di Giovanna PETTI BALBI in preparazione per i Quedemi di Europa moditerranea.

"Anunio de Bntudion Modievalen", 24 (1994) 
stato di Milano, mentre le relazioni finanziario-cambiarie tra Barcellona e Milano passavano attraverso il tramite obbligato di Venezia ${ }^{2}$.

Non si possono tuttavia escludere iniziative precedenti, benché probabilmente di corto respiro, che fanno parte della grande libertà di impresa che caratterizza la mercatura medievale. In particolare la prima metà del Trecento corrisponde all'espansione mediterranea del commercio catalano, legata alle nuove acquisizioni territoriali in Sicilia e in Sardegna: Maiorca, la "porta dell'Africa", in questo periodo raggiunse il culmine della sua prosperita commerciale ${ }^{3}$. Non e quindi casuale che operatori maiorchini si spingessero in area lombarda, favoriti dall'alleanza, in funzione antigenovese, di Pietro IV il Cerimonioso con Venezia e con Milano4. Infatti nel 1349 era a Milano un mercante di Maiorca, Bernardo Egidius, in relazione di affari con vari connazionali ${ }^{5}$. E' inoltre da tenere presente la posizione preponderante di Venezia nel Trecento quale porto di imbarco per le esportazioni milanesi; le navi veneziane avevano in Maiorca il principale scalo mediterraneo sull'itinerario Fiandre-Inghilterra ${ }^{6}$. Ed era stata proprio la volontà di sottrarsi all'ingombrante egemonia veneziana a suggerire l'alleanza dei Visconti di Milano con la Corona d'Aragona'. Neppure nella prima metà del Quattrocento, durante i decenni di alleanza e di amicizia personale fra Alfonso il Magnanimo e Filippo Maria Visconti si ebbe una significativa presenza di mercanti catalani, mentre invece i sudditi

\footnotetext{
${ }^{2}$ Per quanto sopra rimando al mio lavoro Mercanti lombardi fra Barcellona e Valenze nel basso medioevo, Bologna, 1982.

${ }^{3} \mathrm{~V}$. La sintesi in Jesùs LALINDE ABADlA, La Corona de Aragón en el Mediterraneo medieval (1229-1479), Zaragoza, 1979, p. 218 e ss.

"Infatti nel 1347 Pietro IV aveva inviato in proposito a Milano un'ambasceria. Per quanto sopra v. Alberto Boscolo, Catalani nel Medioevo, Bologna, 1986, p. 152.

Ill mercante era nominato procuratore da un altro maiorchino, condottiero al servizio di Giovanni Visconti, signore di Milano, per riscuotere vari crediti che quest 'ultimo vantava per prestiti concessi a connazionali (Patrizia MAINONI, Economia e politica nella Lombardia medievale. Da Bergamo a Milano fra XIII e XV secolo, Gribaudo ed., Cavallermaggiore (CN) 1994, p. 156).

${ }^{6}$ MAINONI, Economia e politica, p. 193.

'Le relazioni politiche fra $\mathrm{i}$ Visconti di Milano e la Corona d'Aragona sono state oggetto di una serie di indagini che hanno posto in luce come, a partire dal momento stesso della completa affermazione della dinastia, negli anni Trenta del XIV secolo, i signori avessero tentato in vari modi di inserirsi nel numero delle potenze mediterranee rivendicando prima la Sardegna, poi imsignorendosi di Genova e infine, persa la città ligure, perseguendo la carta di un'alleanza matrimoniale nei confronti della Sicilia (Gigliola SOL.DI RONDININI, Saggi di storia e storiografia visconteo-sforzesche, Bologna 1984, pp. 86-90; BosCOLO, Catalani, p.68 e i relativi rimandi bibliografici).
} 
del duca di Milano godevano in Catalogna di una posizione di deciso favo$\mathrm{re}^{8}$. Questa situazione si puo spiegare non tanto per il minore sviluppo delle operazioni mercantili catalane, perché anche i lombardi, salvo poche eccezioni, erano ben lontani dall'ampiezza delle ditte toscane, quanto per $\mathrm{i}$ diversi interessi economici dei mercanti catalani, la cui attività era stata inizialmente legata ai trasporti marittimi e solo successivamente si era estesa alla finanza e alla produzione dei panni. Invece quando Napoli entro a fare parte della Corona d'Aragona, e soprattutto in epoca sforzesca, la situazione sembra mutare in quanto in questo periodo alcune ditte catalane fecero perno sul Regno di Napoli per allacciare rapporti diretti con la Lombardia.

Nel secondo Quattrocento il mercato milanese appare caratterizzato da un graduale cambiamento rispetto all'epoca precedente, in parte riconducibile alle trasformazioni generali dell'economia' $\mathrm{e}$ in parte all'evoluzione delle strutture politiche del ducato dopo la presa di potere da parte della nuova dinastia degli Sforza. In particolare lo stato di Milano aveva attraversato un momento di crisi dopo la morte del duca Filippo Maria Visconti (1447) che aveva designato suo erede Alfonso il Magnanimo re d'Aragona ed ora anche di Napoli: la mancata successione aragonese aveva rallentato le relazioni commerciali fra Lombardia ed area catalana, che, come detto, avevano conosciuto il momento di maggiore intensità proprio durante l'intesa di Filippo Maria con il sovrano. Francesco Sforza, tuttavia, cui importava consolidare un potere che non aveva giustificazioni dinastiche, ricercava l'appoggio di Alfonso il Magnanimo e si faceva promotore di una politica matrimoniale nei riguardi del regno di Napoli che sarebbe proseguita per tutto il Quattrocento ed avrebbe stretto insieme, pure con alterne vicende legate al contesto della politica degli stati italiani, le sorti del ducato di Milano e del Regno meridionale ${ }^{10}$. Pegno dell'alleanza fu la concessione del ducato di Bari a Sforza Maria, figlio di Francesco Sforza, nel 1464 e,

\footnotetext{
${ }^{8}$ Patrizia MaINONI, Mercanti lombardi, p. 73 e nota 174.

${ }^{9}$ Legate soprattutto, ma non solo, all'espansione del setificio: un quadro a grandi linee ancora valido è quello delineato in Gino BARBIERI, Economia e politica nel ducato di Milano, 1385-1535, Milano, 1938.

${ }^{10} \mathrm{G}$. SOI.DI RONDININI, Saggi di storia e storiografia, p. 106 e ss. Vennero infatti conclusi nel 1457 i contratti per due matrimoni, quello di Ippolita Sforza con Alfonso d'Aragona, e di Sforza Maria con Elconora d'Aragona, cioé fra due figli di Francesco Sforza e due figli di Ferrante; tuttavia solo il primo ebbe effettivamente luogo, nel 1465.
} 
dopo la morte di questi, a Ludovico Maria suo fratello, futuro duca di Milano $^{11}$. Il legame con Milano significd anche la concessione di una serie importante di privilegi mercantili, che ponevano i lombardi sullo stesso piano dei veneziani e dei mercanti autoctoni, sia per Bari sia per l'intero regno di $\mathrm{Napoli}^{12}$. Manca tuttavia un'analisi documentata del rapporto commerciale che si instauro con il Meridione, testimoniato, oltre che dalla concessione dei privilegi, dall'esistenza della nazione dei Lombardi a Napoli e, ancora di più, dal consolato mercantile a Bari ${ }^{13}$. Si era avuta, in sintesi, l'apertura di un nuovo mercato, il cui raggio di azione non si limitava a Napoli, ma risaliva verso Roma e Ancona, cioe lungo l'itinerario verso la capitale. In alcuni settori dell'armamento si ebbero tra Milano e Napoli scambi di forniture su cui si dovrebbe meglio indagare, anche nella direzione di una partecipazione personale dei regnanti ad imprese commerciali ${ }^{14}$.

La conquista aragonese del regno di Napoli aveva portato ad un crescente inserimento della finanza catalana nel Regno meridionale, che nel secondo Quattrocento appare controllato, dal punto di vista economico, dai toscani per quanto riguarda la finanza internazionale, dai catalani per la finanza pubblica e le operazioni su piazza ${ }^{15}$. Mario Del Treppo ha bene dimostrato la crescente penetrazione delle compagnie mercantili catalane in

\footnotetext{
"Malgrado il patto nuziale con Eleonora venisse sciolto nel 1472 il ducato di Bari venne riconfermato agli Sforza, che lo governavano tramite funzionari milanesi. In cambio del matrimonio mancato si conclusero invece gli accordi per le nozze di Giangaleazzo Sforza, erede del ducato di Milano, con Isabella figlia del duca di Calabria e di Ippolita Sforza. Per quanto sopra si v. il vecchio, ma solido lavoro di enudizione di Nicola FERORELU, Il Ducato di Bari sotto Sforza Maria e Ludovico il Moro (da documenti inediti del R. Archivio di Stato, dell'Ambrosiana e della Trivulziana di Milano), Milano, 1914.

${ }^{12} \mathrm{~V}$. ora Francesco STRAZZULL, I Lombardi a Napoli sulla fine del'400, Napoli, 1992, pp. 27-34; per Bari anche Giosue MUSCA, Storia di Bari dalla conquista normanna al ducato sforzesco, Bari, 1990, pp. 155-156.

${ }^{13}$ Ettore Verga, La Camera dei Mercanti di Milano, Milano, 1914, nuova ed. 1978, pp. 51 e 8s.; v. anche le indicazioni in Il giomale del Banco Strozzi di Napoli (1473), a cura di Alfonso LEONE, Napoli, 1981, pp. 614-615.

${ }^{14}$ Bbidem, p. 259, posta relativa a 1.000 corazze vendute alla Corte napoletana "secondo la forma di cierti capitoli"; la provenienza non e indicata, ma non d una coincidenza che poco più di un mese prima il duca di Milano Galeazzo Maria Sforza avesse commissionato "doa millia armature italiane" facendo lavorare in esclusiva tutti i maestri armaioli di Milano (ASMi, Sforzesco, cart.223, 1473 febbraio 23).

${ }^{15}$ Mario DEL TREPPO, I mercanti catalani e l'espansione della Corona d'Aragona nel secolo XV, Napoli, 1972, trad. catalana Els mercaders catalans $i$ l'expansio de la Corona catalano-aragonesa al segle XV, Barcelona, 1976, p.171 e ss.; ID., Napoli e la Corona d'Aragona: appunti per un bilancio storiografico, in Fonti e cronache italo-iberiche del basso medioevo. Prospettive di ricerca, Firenze, 1984, pp. 48-50.
} 
concomitanza, e in ragione, dell'impresa italiana di Alfonso, con lo stretto legame fra il finanziamento della spedizione e i successivi interventi nei traffici commerciali con il Regno, soprattutto la vendita di panni catalani e l'importazione del sale di Ibiza, rapporti che non ebbero termine con la rivolta di Barcellona e la difficoltosa successione di Ferrante nel Mezzogiorno d'Italia. Nella seconda metà del XV secolo il Regno divenne una tappa necessaria nei circuiti finanziari europei, sempre più omogenei e integrati fra Barcellona, Valencia, Roma, Napoli, Firenze, Avignone e Venezia.

La presenza di rappresentanze di ditte catalane a Milano va ricondotta quindi ad un duplice ordine di questioni, l'uno relativo a rapporti di queste ultime con ditte milanesi, l'altro a iniziative in qualche modo dovute all'alleanza dei duchi di casa Sforza con i sovrani aragonesi di Napoli. Non e tuttavia possibile scindere $i$ due aspetti, che rimandano ad operazioni finanziarie trattate dai mercanti-banchieri con le Corti; se inoltre, di norma, secondo il costume delle "nazioni" mercantili, gli operatori agivano appoggiandosi reciprocamente e non servendosi di forestieri, nel secondo Quattrocento si osserva una più spiccata tendenza verso il superamento di questi limiti ed al diffondersi di procure e di patti societari fra mercanti di diversa nazionalità.

Un primo esempio, e il più documentato, non a caso è quello di una ditta di Valencia, la piazza iberica il cui ruolo commerciale aveva conosciuto un forte sviluppo nel corso del Quattrocento, cioe l'azienda degli eredi di Martin Ruiz (o Roiz). Sarebbe interessante fare risalire i rapporti mercantili dei Ruiz con i mercanti milanesi alla prima metà del XV secolo, quando Martin Roiz di Valencia fornl grossi quantitativi di lana ai rappresentanti delle compagnie lombarde qui presenti ${ }^{16}$. Nella seconda metà del Quattrocento gli eredi Ruiz erano i commissionari a Valencia dell'azienda di Ambrogio Ruffini di Milano, un'impresa mercantile attiva dalla prima metà del Quattrocento a Bruges, a Londra, a Valencia e a Barcellona ${ }^{17}$. I Ruffini tuttavia negli anni Ottanta si trovavano in cattive acque: nel 1483 infatti Ambrogio Ruffini ed i nipoti diedero procura ai

\footnotetext{
${ }^{16}$ MAINONI, Mercanti lombardi, p. 75 e nota 195.

${ }^{17}$ Patrizia MAINONI, $L$ 'attivild mercantile e le casate milanesi nel secondo Quattrocento, in Milano nell'etd di Ludovico il Moro, II, Milano, 1983, p. 579.
} 
nobili Consalo Egidius e Martino Egidius, entrambi di Valencia, di riscuotere i crediti loro spettanti, anche nei confronti di altre ditte lombarde ${ }^{18}$.

I Ruiz invece, una casata di conversos valenzani che vanno annoverati fra le maggiori imprese mercantili della città, corrispondenti della filiale Medici di Venezia ${ }^{19}$, con Roma e Bologna attraverso i banchi dei Pazzi e degli Spannocchi e con Siviglia tramite la compagnia genovese, con base appunto nella città atlantica, dei Gentile ${ }^{20}$, avviarono l'anno successivo l'apertura di una rappresentanza a Milano: infatti nel 1484 il re Ferdinando di Castiglia raccomandava al duca di Milano Juan Ferragut, inviato a commerciare in questa città dalla ditta valenzana ${ }^{21}$.

Gli interessi mercantili in Italia dei Ruiz si intrecciavano con le crescenti difficoltà cui andavano incontro i conversos nella Spagna dei Re Cattolici, soprattutto dopo l'impianto dell'Inquisizione a Valencia, nel 1481, che esercito una durissima repressione contro i discendenti degli ebrei convertiti, di cui è indice il privilegio reale ottenuto nel 1488, pagato a caro prezzo, che tutelava le proprietà degli eredi dei condannati ${ }^{22}$. I Ruiz, nella persona di Gonsalvo e Gil Ruiz e di Juan Ferragut, si crearono una via di fuga riuscendo ad ottenere, nel 1486, la cittadinanza milanese per intervento di Simonot Bellprat, già tesoriere di Ferrante d'Aragona ed ora oratore del re di Napoli presso il duca di Milano ${ }^{23}$. Infatti nel 1492, accusati di eresia, riuscirono a fuggire in $\mathrm{Italia}^{24}$, anche se $\mathrm{i}$ loro beni furono confiscati e ancora nel 1496 gli eredi di Ambrogio Ruffini avanzavano formale petizione a Ferdinando il Cattolico di poter recuperare cio che era loro dovuto per la vendita di mercanzie inviate da Milano, da

\footnotetext{
${ }^{18}$ ASMi cart. 1686, 1483 aprile 21.

${ }^{19}$ Enrique CRUSELIES, "Todo es cerrazón y noche". La sociedad urbana valenciana en la encrucijada a los tempos modemos, in "Revista d'Història Medieval. València a la Mediterrania medieval", 3 (1992), p. 153.

${ }^{20}$ David IGUAI. LUIS, Valencia $i$ Sevilla en el sistema econolnico genovés de finales del siglo XV, in "Revista d'Història Medieval. València i la Mediterrània medieval", cit., pp. 91, 110.

${ }^{21}$ Documentos sobre las relaciones internacionales de los Reyes Catolicos, a cura di Antonio de la TORRF, Barcelona, 1949, n. 36 (1482), rapporti con i Medici, e n.115 (1484).

${ }^{22}$ Jacqueline GUIRAL-HADZIIOSSIF, Valence, port méditerranéen au XVe siècle (14101525), Paris, 1986, p. 357. Sul tema v. S. HAIJCZER, Inquisition and Society in the Kingdom of Valencia, 1478-1834, Berkeley, 1990 e le indicazioni citate in CRUSFILLS, op. cit., pp. 135-141.

${ }^{23}$ ASMi, Panigarula, n. 11 , p. 553 e ss.

${ }^{24}$ D. IGUAI. LUIS, op.cil., p.91.
} 
stabilirsi sulla base di cio che risultava dai mastri della ditta, rimasti a Valencia ${ }^{25}$. La situazione personale dei Ruiz dovette di li a poco migliorare perché nel 1498 Martino Ruiz fu Martino, che si era stabilito a Milano, ordino suo procuratore Giovanni Ruiz fu Gonsalvo, suo nipote, per ricevere le merci loro spettanti nel regno di Castiglia, in Aragona, a Valencia e in Catalogna ${ }^{26}$.

L'espansione delle aziende iberiche nella direzione della Lombardia grazie ai legami politici ed economici con la corte aragonese di Napoli e inoltre evidente nel caso di un'importante azienda di Barcellona, ma con sede a Napoli, quella dei fratelli Francesco e Raffaele de Besalü ${ }^{27}$. I Besalù, che le poste del libro del banco Strozzi di Napoli ricordano impegnati in numerosissime operazioni mercantili-bancarie, oltre che nell'esportazione del sale di Ibiza $^{28}$, erano grandi finanziatori della corte regia, di cui Del Treppo ricorda nel 1482 un credito di $\mathbf{4 0 . 0 0 0 ~ d u c a t i . ~ N o n ~ m e r a v i g l i a ~ q u i n - ~}$ di se nel 1486 i Besalù abbiano ottenuto dal duca di Milano un salvacondotto per venire nel dominio con merci, beni e gioielli di qualsiasi genere, unitamente ad una littera contra debitores della durata di due anni: il privilegio, che sottolinea l'esistenza di interessi già consolidati nel ducato sforzesco, copriva non solo i titolari ma anche gli otto soci della compagnia ${ }^{29}$.

Come si pud osservare, la fonte che ha fornito il maggior numero di indicazioni è costituita da salvacondotti o da atti emanati dalle cancellerie: documenti ufficiali che non consentono di datare effettivamente l'attività mercantile, che poteva essere assai precedente, né tanto meno di quantificarla. Ruiz e Besalù erano aziende ad alto livello, legate da rapporti finanziari con le stesse Corti: e possibile che, sulla direttrice dei traffici fra nord e sud d'Italia e della mediazione genovese si inserissero altre ditte catalane ${ }^{30}$.

${ }^{25}$ Documentos sobre relaciones intemaciomales, vol. 5, 1961, n. 207 (anno 1496): il testo cita gli eredi di Ambrogio de Rosinis, ma l'identificazione con i Ruffini (de Rufinis, de Rofinis) mi pare assai probabile. Cfr. la citazione in BOSCOLO, Catalani, p. 138.

${ }^{26}$ ASMi, F.N., cart. 3443, 1498 dicembre 6.

${ }^{27}$ DEL TREPPO, Els mercaders, p. 215, 216 ecc.

${ }^{28} \mathrm{Nel}$ mastro del banco Strozzi del 1473 le citazioni sono frequentissime: ad es. p. 149.

${ }^{29}$ ASMi, Registri ducali $n .91$, fo. 150-151. Nell'atto non sono nominati i soci.

${ }^{30}$ Del Treppo ricorda, sullo scorcio del XV secolo, la compagnia fondata dal tesoriere generale di Spagna Gabriel Sánchez e dal batlle di Catalogna, Joan Sarriera, che importava a Napoli e in Sicilia panni catalani, seta, olio, spezie, con navi proprie, ed aveva relazioni commerciali con Genova e con Milano (DEL TRFPPO, Els mercaders, p. 531 e nota 140). 
E' tuttavia riduttivo limitare allo scambio interno alla penisola italiana gli interessi delle compagnie, che avevano procuratori e soci sulle piu importanti piazze iberiche e specialmente a Siviglia. Mentre, fra i prodotti milanesi diretti all'area iberica, le armi conservavano un ruolo di primissimo piano ${ }^{31}$, e bene possibile che fra gli oggetti dei traffici diretti a Milano la seta spagnola godesse di una domanda sostenuta, come attestano diverse indicazioni dell'ultimo Quattrocento ${ }^{32}$; una mercanzia di probabile provenienza dalle isole atlantiche era lo zucchero, che, ad esempio, era importato da una ditta milanese che aveva un'ampia rete di relazioni in Spagna, quella dei Litta ${ }^{33}$. Anche i Litta entrarono direttamente in societa con mercanti iberici, come nel caso della compagnia per il commercio dei libri a stampa fondata nel 1482 a Valencia fra Alberto Litta e Francesco Dametzana barreter, gia mercante di libri 'vulgo dicti de emprunta ${ }^{34}$.

Cio che emerge nettamente da quanto sopra detto e la trasformazione verificabile non solo a proposito di itinerari o di merci, ma nell'atteggiamento stesso dei mercanti. Si hanno ora anche società a carattere internazionale, che sono state particolarmente studiate a proposito dell'altro grande polo dei traffici fra Atlantico e Mediterraneo, Siviglia: genovesi con spagnoli, spagnoli con fiorentini e genovesi' ${ }^{35}$, milanesi con valenzani: la "piattaforma internazionale puntellata sul denaro" che, grazie proprio al consolidarsi di grandi Stati monarchici, "parrebbe ancor più prepotentemente proporsi al di sopra delle compagini statuali...una sorta di sovrastruttura inglobante, tenuta insieme da quel mastice incomparabile rappresentato dall'interesse economico" ${ }^{36}$.

${ }^{31}$ MAINONI, Mercanti lombardi, pp. 88-89; ASMi,F.N., cart. 1945, 1473 ottobre 25.

${ }^{32}$ ASMi, F.N., cart. 3440, 1480 gennaio 29, cart. 1686, 1483 novembre 25, cart. 3440, 1491 gennaio 22, cart. 3443, 1497 settembre 27 ecc., "seta spanyarda".

${ }^{33}$ ASMi, cart.1223, 1471 aprile 4. Sui Litta v. MAINONI, Mercanti, pp. 120-121.

${ }^{34}$ Valencia, Archivo del Patriarca, prot. n. 1155. La societa, della durata di quattro anni, venne sciolta nel 1486. Sul ruolo dei Litta quali ambasciatori in Spagna v. Giuliana FANTON, Milano e Spagna alla fine del Quattrocento: le lettere di Francsco Litta a Ludovico il Moro, in "Quademi di letterature iberiche e ibero-americane", 18-20 (1993), pp. 5-28.

${ }^{35}$ Enrique OTTE, Il ruolo dei Genovesi nella Spagna del XV e XVI secolo, in La repubblica intemazionale del denaro tra XV e XVII secolo, a cura di Aldo DE MADDALENA o di Hermann KEIUPNBENZ, Bologna, 1986, p. 23.

${ }^{36}$ Aldo DE MADDALENA, La repubblica insemazionale del denaro :un ipotesi infondase 0 una tesi sostenibile?, in La repubblica insemazionale del denaro, p. 8. 


\section{RÉSUMÉ}

L'étude de la présence des marchands étrangers dans l'Europe du Moyen Age et de la Renaissance doit considérer leurs liens avec le pouvoir politique: ainsi, quand on trouve mention a Milan de marchands du Royame d'Aragon, même si elles sont peu importantes, si on peut analyser l'appui politique dont ils ont joui. Quelques marchands de Majorque étaient a Milan durant le milieu du XIVe siecle, quand le roi d'Aragon était allié avec Milan et Venise contre Gênes, mais les Catalans, engagés dans les trafics maritimes, ne semblent pas s'être intéressés a l'intérieur de l'Italie du Nord jusqu'à la deuxième moitié du XVe siècle, quand la conquête du Royaume de Naples par Alfonso el Magnánimo permit la pénétration de plusieurs marchands-banquiers catalans. Certaines parmi les plus grandes firmes eurent des rapports d'affaires avec des maisons marchandes de Milan et envoyèrent aussi sur place des représentants. Elles étaient favorisées en cela par l'alliance, renforcée par les liens de mariage, entre Ferdinando I et les Sforza ducs de Milan. Un exemple bien documenté est celui de la firme valencienne des héritiers de Martin Ruiz: les Ruiz obtinrent le privilegium civilitatis de Milan en 1486 par l'intervention de Simonot Bellprat, qui avait été trésorier du roi de Naples et était en ce temps-là ambassadeur à Milan. Ils s'étaient enfuis de Valence parce qu'ils étaient conversos et s'établirent à Milan.

\section{SUMMARY}

The study of temporary settlements of foreign merchants in medieval and Renaissance Europe needs also to consider their relations with political power; in this context if we found data about traders of the Kingdom of Aragon in Lombardy, however few, they would be of importance if they helped us to analyse what political support the merchants could expect and how it worked.

We have news of Majorcan merchants in Milan in the middle of the fourteenth century, when the King of Aragon allied with Milan and Venice allied against Genova; nevertheless, more occupied in maritime trade, Catalan merchants didn't extend their business range towards the interior of northern Italy till the second half of the fifteenth century, when the con- 
quest of the Kingdom of Naples by Alfonso el Magnánimo brought many Catalan merchant-bankers to the southern capital. Some of those firms, the bigger ones, had business relations with Milanese firms and also sent representatives to Milan, encouraged by the alliance, consolidated by marriage ties, between Ferdinando I, king of Naples, and the Sforza dukes of Milan. A well documented example is that of the Valencian firm of the heirs of Martin Ruiz, who obtained the privilegium civilitatis of Milan in 1486 through the intervention of Simonot Bellprat, treasurer of the King of Naples and now ambassador et the Sforza Court; after a few years the Ruiz had to flee from Valencia because they were conversos, and chose to settle in Milan. 